\title{
Log-Normal Model is the Best Fitted Model for Duration from Chest Pain to Coronary Artery Disease Diagnosis: An Outcome of Retrospective Cross Sectional Study
}

\author{
Mehwish Hussain \\ Department of Research \\ Dow University of Health Sciences, Karachi, Pakistan \\ mehvish.hussain28@gmail.com \\ Nazeer Khan \\ Department of Research \\ Jinnah Sindh Medical University, Karachi, Pakistan \\ nazeerkhan54@gmail.com \\ Mudassir Uddin \\ Department of Statistics, University of Karachi, Karachi, Pakistan \\ mudassir2000@hotmail.com
}

\begin{abstract}
Cox proportional hazard model is the widely used technique for studying duration data. However, studies showed parametric modeling yields better estimation; though these models are less used due to their complicated application and interpretation. Alongside, the duration from chronic chest pain to the diagnosis of coronary artery disease has not evaluated in the literature. Therefore, this research investigated application of parametric modeling on the current duration while studying outcome from cross-sectional study. Akaike Information Criteria was used to adjudicate different duration models.
\end{abstract}

Keywords: Chest Pain, coronary artery disease, parametric duration analysis, Akaike Information Criteria, log-normal distribution

\section{Introduction}

Coronary artery disease (CAD) is ranked highest among chronic fatal diseases worldwide (Murray and Lopez, 1996). Chest pain is one of the perceptible symptoms for the disease (Bösner et al, 2010; Hussain and Khan, 2011; Hussain et al, 2011). The statistics lie around studying prevalence, risk factors, treatment and hazards of CAD have been sufficiently published (Torpy et al, 2009; Genders and Hunink, 2012). However, hazardous predictors imperiling the shortening of the duration from chest pain to the diagnosis of CAD have not been studied yet (Hussain and Khan, 2011; Hussain et al, 2011). For duration modeling, non-parametric, semi-parametric and parametric methods are used (Kieding et al, 2002; Hussain and Khan, 2011; Hussain et al, 2011). Amongst them, parametric modeling yields good estimate with better interpretation (Morkveld, 2007). Nevertheless, these models are rarely considered to be computed due to two reasons, one is that the distribution of duration must be known and the other reason is that interpretation of estimated coefficient is not much comprehensible (MoghimiDehkordi et al, 2008). This study filled the gap while estimating parametric models for the chronic chest pain in patients who later diagnosed with coronary artery disease. 


\section{Duration Models}

\subsection{Non-Parametric Model}

Cox D. R. was the first statistician who derived a regression model to estimate life tables with censored observation (Cox, 1972). Censored observations are referred as any subject who could not achieve event of interest during specified study duration (Moeschberger and Klein, 2003). The idea was first given in 1958 by Edward Kaplan and Paul Meier in their marvelous paper (Kaplan and Meier, 1958). Cox regression model estimates the hazard of risk factors for disease encountered from the onset of symptoms. In our study we planned to estimate hazardous factors for coronary artery disease among the patients when they encountered their first chest pain. Cox proportional hazard model for the duration of CAD is defined as

$$
h(t / d)=h_{0}(t) \exp \left(d_{1} \beta_{1}+d_{2} \beta_{2}+d_{3} \beta_{3}+\cdots+d_{k} \beta_{k}\right)
$$

Here, $h(t / d)$ is hazard of experiencing CAD at certain time ' $t$ ' condition to values of duration from their first chest pain $\boldsymbol{d}=\left(d_{1}, d_{2}, d_{3}, \ldots, d_{k}\right)$. The baseline hazard function is mentioned here as $h_{0}(t)$. The estimates of duration model coefficient $\boldsymbol{\beta}=\left(\beta_{1}, \beta_{2}, \beta_{3}, \ldots, \beta_{k}\right)^{T}$ are derived by the likelihood function given as: $\boldsymbol{L}(\boldsymbol{\beta})=$ $\prod_{r \in C} \frac{\exp \left(\boldsymbol{\beta}^{T} \boldsymbol{x}^{j_{r}}\right)}{\left\{\sum_{j \in R_{r}} \exp \left(\boldsymbol{\beta}^{T} \boldsymbol{x}^{j}\right)\right\}}$

Here $\mathrm{C}$ is the indices of $\mathrm{CAD}$ diagnosis, $\mathrm{R}_{\mathrm{r}}$, is the set of indices of chest pain patients at the risk of having CAD and $j_{r}$ is the index of CAD diagnosis at duration $t_{r}$.

Cox proportional hazard model is considered as semi-parametric model as hazard model coefficient are known (Klienbaum and Klien, 2011). Nevertheless, distribution of time duration appears to be unknown as baseline hazard function in the model is deleted when hazard ratio are computed for the model (Hosmer and Lameshow, 1999; Morkveld, 2007). The interpretation of proportional hazard model is based on hazard ratio (Klienbaum and Klien, 2011). This model produces consistent results with random sampling (Morkveld, 2007). The assumption of proportional hazard implied that hazard ratio among different categories of a factor should not intersect over the duration (Cox, 1972). This assumption is assessed by different methods. One of the methods is to plot negative natural logarithm of natural logarithm of duration function over time duration (Hussain and Khan, 2011). Though, a statistical review on application of survival analysis revealed that only $5 \%$ of studies had checked the underlying assumption of proportional hazards (Altman et al, 1995). Ata et al estimated methods to build such models when assumption of proportional hazards does not hold (Ata et al, 2008).

\subsection{Parametric Model}

Parametric duration models strictly depend on distributional property of time duration (Kleinbaum and Klein, 2005). These models can be referred as proportional hazard (PH) and accelerated failure time (AFT) models (Hayat et al, 2010). Though, most of the parametric distributions hold accelerated failure time property (Gardiner, 2010; Hayat et al, 2010). Hence, these models are put under consideration in this study. The accelerated failure time model are expressed in terms of linear function of natural 
logarithm of duration, such as $\ln \left(d_{j}\right)=x_{j} \beta_{j}+\ln \left(e_{j}\right)$. Here, $d_{j}$ is the duration from chronic chest pain to the diagnosis of $\mathrm{CAD}, x_{j}$ represents vector of explanatory variables, $\beta_{j}$ is defined the regression coefficient and $e_{j}$ indicates the residual vector of the regression model. Due to possessing distributional property, residuals can be computed which intuitively follows normal distribution (Hosmer et al, 2008). Unlike proportional hazard models, the interpretation of accelerated failure time models is based on time duration (Rodriguez, 2005; Hayat et al, 2010; Klienbaum and Klien, 2011).

These models produces consistent results where random sampling cannot persists such as in current duration analysis data (Morkveld, 2007). Both situation of non-random sampling and current duration data are frequent in medical settings (Kieding et al, 2002). The parametric distributions used for estimating duration from the onset of chest pain to the diagnosis of coronary artery diseases are described here.

\subsubsection{Exponential Duration Model}

Exponential distribution is the simplest time duration distribution with constant hazard. If $f(t)=\lambda e^{-\lambda t}$ defines as exponential duration distribution then its duration function is defined as $D\left(T_{i} / \beta\right)=1-F\left(T_{i} / \beta\right)$. As, it is known that $F\left(T_{i} / \beta\right)=1-e^{-\lambda t}$, thus the duration function equated to be $D\left(T_{i} / \beta\right)=e^{-\lambda t}$. The hazard function can be found out by using the property of $f(t)=h(t) D\left(T_{i} / \beta\right)$. Hence, hazard function of exponential distribution would be $h(t)=\lambda$.

Thus, exponential duration model would be defined as:

$$
\ln \left(d_{j}\right)=x_{j} \beta_{j}
$$

\subsubsection{Weibull Duration Model}

Weibull distribution is the expansion of exponential distribution while incorporating the scale parameter. If Weibull duration density has the form $f(t)=\lambda p t^{p-1} e^{-\lambda t^{p}}$ which directs to produce $F(t)=1-e^{-\lambda t^{p}}$ then the duration function of the distribution would be $D\left(T_{i} / \beta\right)=e^{-\lambda t^{p}}$. Thus, using property $f(t)=h(t) D\left(T_{i} / \beta\right)$ the hazard function of Weibull distribution would be $h(t)=\lambda p t^{p-1}$ Here, $\lambda$ is a positive scale parameter and $\mathrm{p}$ is positive shape parameter.

Thus, weibull duration model would be defined as:

$$
\ln \left(d_{j}\right)=x_{j} \beta_{j}+p \log \lambda+\log p+\text { slope } p^{-1}
$$

\subsubsection{Log-Logistic Duration Model}

Log-logistic, also called as Fisk distribution in finance and economics (Lemonte, 2014), is better to apply when there is slow decrease in curve after reaching the peak point (Hayat et al, 2010). The density function with $\lambda$ as scale parameter and $p$ as shape parameter has form $f(t)=\frac{\left(p^{p} / \lambda\right)(t / \lambda)^{p-1}}{\left[1+(t / \lambda)^{p}\right]^{2}}$, yields the duration function as $D\left(T_{i} / \beta\right)=$ $\frac{1}{1+(t / \lambda)^{p}}$ and hazard function as $H(t)=\frac{(p / \lambda)(t / \lambda)^{p-1}}{1+(t / \lambda)^{p}}$. 
Thus, log-logistic duration model would be defined as:

$$
\ln \left(d_{j}\right)=x_{j} \beta_{j}+p \log \lambda+\log p
$$

\subsubsection{Log-Normal Duration Model}

When average values are low and variances are high then such positive skewed distribution conforms with log-normal duration distribution (Hayat et al, 2010). Thus, if the distribution with the form $f(t ; \lambda, p)=\frac{1}{t p \sqrt{2 \pi}} e^{-\frac{(\mathrm{lnt}-\lambda)^{2}}{2 p^{2}}}$ follows log-normal distribution then the duration function of the same distribution is described as $D\left(T_{i} / \beta\right)=1-$ $\phi\left(\frac{\ln (t)-\lambda}{p}\right)$ and hazard function would be found out as $H(t)=\frac{f(t)}{D(t)}$.

Hence, log-normal duration model would also be defined as:

$$
\ln \left(d_{j}\right)=x_{j} \beta_{j}+p \log \lambda+\log p
$$

\subsection{Adjudicating between Different Duration Models}

To adjudicate the most appropriate parametric model for the duration from chronic chest paint to the diagnosis of coronary artery disease, Akaike Information Criteria (AIC) was used (Akaike, 1974). The AIC value was computed using formula; AIC $=-2 \mathrm{x}$ (loglikelihood $)+2(\mathrm{k}+1+\mathrm{m})$. here $\mathrm{k}$ defines the number of independent variables in the model excluding the constant term, $\mathrm{m}$ defines number of parameters in the model (Hayat et al, 2010). Among the four parametric models i.e. exponential, weibull, log-logistic and log-normal, the model which produced minimum AIC value was considered as good fitted model for the data.

\subsection{The Application on Coronary Artery Disease}

The data were obtained from 5 years epidemiological study on Coronary Artery Disease in Saudi (CADIS) population (Al Nouzha et al, 2004). The study was conducted during 1995 - 2000 on 17,232 native subjects residing in the country at the time of study. Multistage sampling was adopted to recruit participants from urban and rural areas of all 14 regions of the kingdom. The inclusion criteria were set to be subjects of both genders within $30-70$ years of age. A detailed questionnaire was constructed which covered different aspects of demography, history and clinical findings of sampled individuals. Well-trained doctors carried out related clinical diagnosis and physical examination of participants. Patients were asked about the time of first onset of their chest pain and then the duration was noted down. The criteria for the diagnosis of coronary artery disease were made by any one of the three manifestations which are 1) clinical assessment by trained physician, 2) record of previous myocardial infarction and 3) findings from electrocardiography of participant.

For performing duration analysis in Stata v. 12.0, certain preliminary steps were performed. First of all, the CADIS data were converted from cross sectional to time duration. The status of the diagnosis of coronary artery disease was set as event variable. Duration from first onset of chest pain to the study period was set as time variable. Those subjects who encountered chest pain in past but had not experienced CAD were kept as censored observations. Kaplan-Meier curve was plotted to observe shape of distribution 
CAD duration. Male Gender, triglyceride nitrate (> $2.3 \mathbf{~ m m o l} / \mathbf{L})$ and high density lipoprotein (> $\mathbf{0 . 7 5} \mathbf{~ m m o l} / \mathbf{L}$ ) were the predictors used in duration model. Proportional hazard assumption was checked by Shoenfeld residuals test. The $p$ value of each predictor was more than $\mathbf{0 . 0 5}$, therefore, this assumption was considered to be fulfilled. Cox regression and parametric models of exponential, weibull, log-logistic and log-normal were built. Akaike information criterion was not built-in function in Stata. Hence, it was manually manipulated using above defined formula.

\section{Results}

Out of 17,232 participants, $24 \%(n=4130)$ encountered chest pain. Among those, 1,182 patients were diagnosed with coronary artery disease. The average duration from chest pain to the diagnosis of CAD was 5 years with minimum duration was 1 year and maximum was 40 years. Within 1 year, $12 \%$ of the patients with chest pain experienced CAD. Within 5 years, this proportion projected to $57 \%$. After 10 years, there is a gradual decrease in the distribution of the same which lasted to 40 years (Fig. 1).

Among 4,130 patients with chest pain 2,220 (52.75\%) were male and 1,910 (46.25\%) were female. High density lipoprotein (HDL) was higher in 1,414 (34.24\%) patients. Triglyceride nitrate (TGN) was more than $2.3 \mathrm{mmol} / \mathrm{L}$ in $87(2.1 \%)$ of the patients.

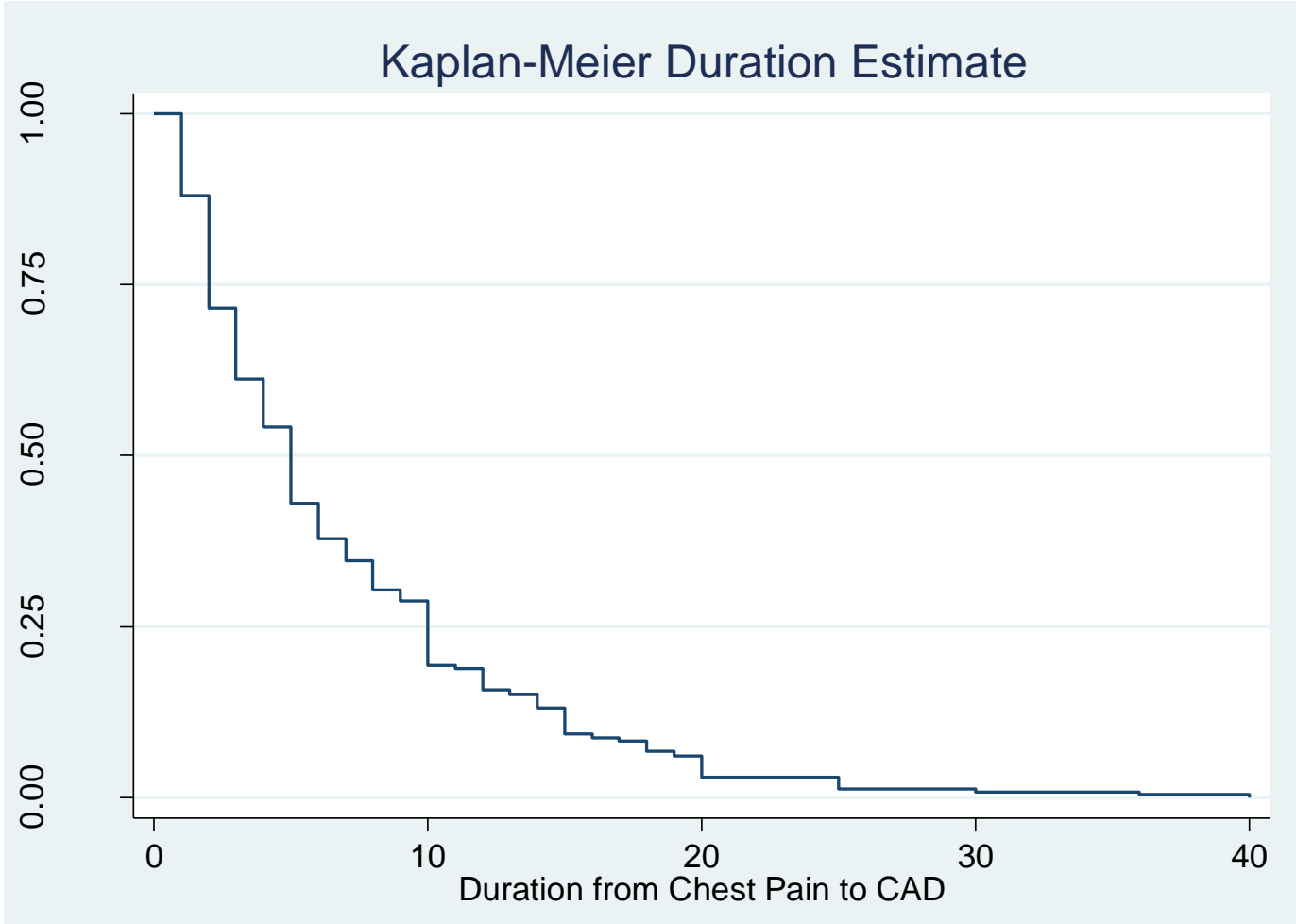

Fig. 1: Kaplan-Meier Curve showing Distribution of Duration from Chest Pain to CAD 
Cox regression analysis revealed that male had 1.173 times higher hazard of having CAD after the onset of chest pain as compare to female. High HDL caused $30.2 \%$ decrease in hazard of having CAD as compare to those patients who had low HDL. Similarly, patients with TGN more than $2.3 \mathrm{mmol} / \mathrm{L}$ had 53.2\% reduced hazard as compare to those who had low TGN. The test of significance showed that these three variables were significant predictors. The $\mathrm{p}$ value obtained from likelihood ratio test also indicated goodness of fit of the models. The AIC values of the models using gender, HDL and TGN as predictors were calculated as 17214.56, 17102.83 and 16478.95 respectively (Table 1). The PH assumption of these variables was fulfilled as Schoenfeld test produced $p$ values as 0.5214 for gender, 0.9589 for HDL and 0.369 for TGN.

Table 1: Cox Model for Duration from Chronic Chest Pain to the Diagnosis of CAD

\begin{tabular}{|c|c|c|c|c|c|c|c|c|c|c|}
\hline Factor & $\begin{array}{c}\text { Hazard } \\
\text { Ratio }\end{array}$ & $\begin{array}{c}\text { S.E. } \\
(\mathbf{H R})\end{array}$ & $\mathbf{Z}$ & $\mathbf{P}>|\mathbf{z}|$ & \multicolumn{2}{|c|}{$\begin{array}{c}\text { [95\% Conf. } \\
\text { Int }]\end{array}$} & $\begin{array}{c}\text { Log- } \\
\text { Likelihood }\end{array}$ & $\begin{array}{c}\text { Likelihood } \\
\text { Ratio Test }\end{array}$ & $\begin{array}{c}\text { P > } \\
\text { Chi-Sq }\end{array}$ & $\begin{array}{c}\text { AIC } \\
\text { Value }\end{array}$ \\
\hline Male & 1.173 & 0.047 & -2.860 & 0.004 & 1.308 & 1.052 & -8605.280 & 8.200 & 0.004 & 17214.559 \\
\hline $\begin{array}{c}\text { HDL > } \\
\mathbf{0 . 7 5} \\
\mathbf{m m o l} / \mathbf{L}\end{array}$ & 0.642 & 0.118 & -2.40 & 0.016 & 0.446 & 0.922 & -8551.236 & 6.66 & 0.001 & 17106.472 \\
\hline $\begin{array}{c}\text { TGN }> \\
\mathbf{2 . 3} \\
\mathbf{m m o l} / \mathbf{L}\end{array}$ & 0.468 & 0.759 & 2.140 & 0.032 & 0.233 & 0.937 & -8237.477 & 5.980 & 0.014 & 16478.953 \\
\hline
\end{tabular}

The parametric modeling for the duration from chronic chest pain to the diagnosis of coronary artery disease revealed that among the four parametric distributions, log-normal distribution produced the lowest AIC value. Therefore, it can be estimated that the duration from chronic chest pain to the onset of CAD followed log-normal distribution (Table 2).

Table 2: Parametric Models of Duration from Chronic Chest Pain to Diagnosis of CAD

\begin{tabular}{|c|c|c|c|c|c|}
\hline $\mathbf{x}$ & Distribution & Model & P Value & Log-Likelihood Value & AIC Value \\
\hline \multirow{4}{*}{ Gender } & Exponential & $1.167 \mathrm{x}$ & 0.0052 & -2148.35 & 4300.702 \\
\hline & Weibull & $1.211 \mathrm{x}+0.217 \ln (\mathrm{p})+1.243 \mathrm{p}+0.805 \mathrm{p}^{-1}$ & 0.0006 & -2098.55 & 4203.098 \\
\hline & Log-Logistic & $1.592-0.135 x-0.599 \ln (p)+0.549 p$ & 0.0059 & -2059.27 & 4124.547 \\
\hline & Log-Normal & $1.582-0.114 x-0.083 \ln (p)+0.920 p$ & 0.0149 & -2019.56 & 4045.121 \\
\hline \multirow{4}{*}{$\begin{array}{c}\text { HDL > } \\
0.75 \\
\mathrm{mmol} / \mathrm{L}\end{array}$} & Exponential & $0.639 \mathrm{x}$ & 0.0092 & -2122.89 & 4249.780 \\
\hline & Weibull & $0.641 \mathrm{x}+0.212 \ln (\mathrm{p})+1.236 \mathrm{p}+0.808 \mathrm{p}^{-1}$ & 0.0097 & -2075.38 & 4156.760 \\
\hline & Log-Logistic & $1.501-0.3834 x-0.602 \ln (p)+0.547 p$ & 0.0085 & -2032.87 & 4071.740 \\
\hline & Log-Normal & $1.504-0.353 x-0.087 \ln (p)+0.917 p$ & 0.0105 & -1992.97 & 3991.940 \\
\hline \multirow{4}{*}{$\begin{array}{c}\text { TGN > } \\
2.3 \\
\mathrm{mmol} / \mathrm{L}\end{array}$} & Exponential & $0.460 \mathrm{x}$ & 0.0121 & -2053.15 & 4110.30 \\
\hline & Weibull & $0.444 \mathrm{x}+0.213 \ln (\mathrm{p})+1.238 \mathrm{p}+0.808 \mathrm{p}^{-1}$ & 0.0084 & -2006.78 & 4019.551 \\
\hline & Log-Logistic & $1.515-0.563 x-0.603 \ln (p)+0.547 p$ & 0.0349 & -1966.64 & 3939.272 \\
\hline & Log-Normal & $1.518-0.464 x-0.087 \ln (\mathrm{p})+0.917 \mathrm{p}$ & 0.0537 & -1928.49 & 3862.976 \\
\hline
\end{tabular}


Graphical presentation of parametric model exhibited all models appeared to be good fitted to the distribution of duration from chest pain to CAD with median duration of 5 years (Fig. 2). However, the distinct analysis demonstrated that curve from exponential model originated from $80 \%$ of duration function and inclined to $\mathrm{x}$-axis after the duration of 25 years. Weibull distribution displayed the same distribution as exponential. Nevertheless it inclined to $\mathrm{x}$-axis earlier than exponential distribution i.e. at the duration of 20 years. Curves of $\log$ - logistic distribution and $\log$ - normal distribution showed similar pattern of duration. Though, the duration function from $\log -\operatorname{logistic}$ delayed to incline towards horizontal axis. Log - normal distribution was the only distribution which displayed the same statistical distribution as of original duration evaluated by KaplanMeier curve (Fig, 1).
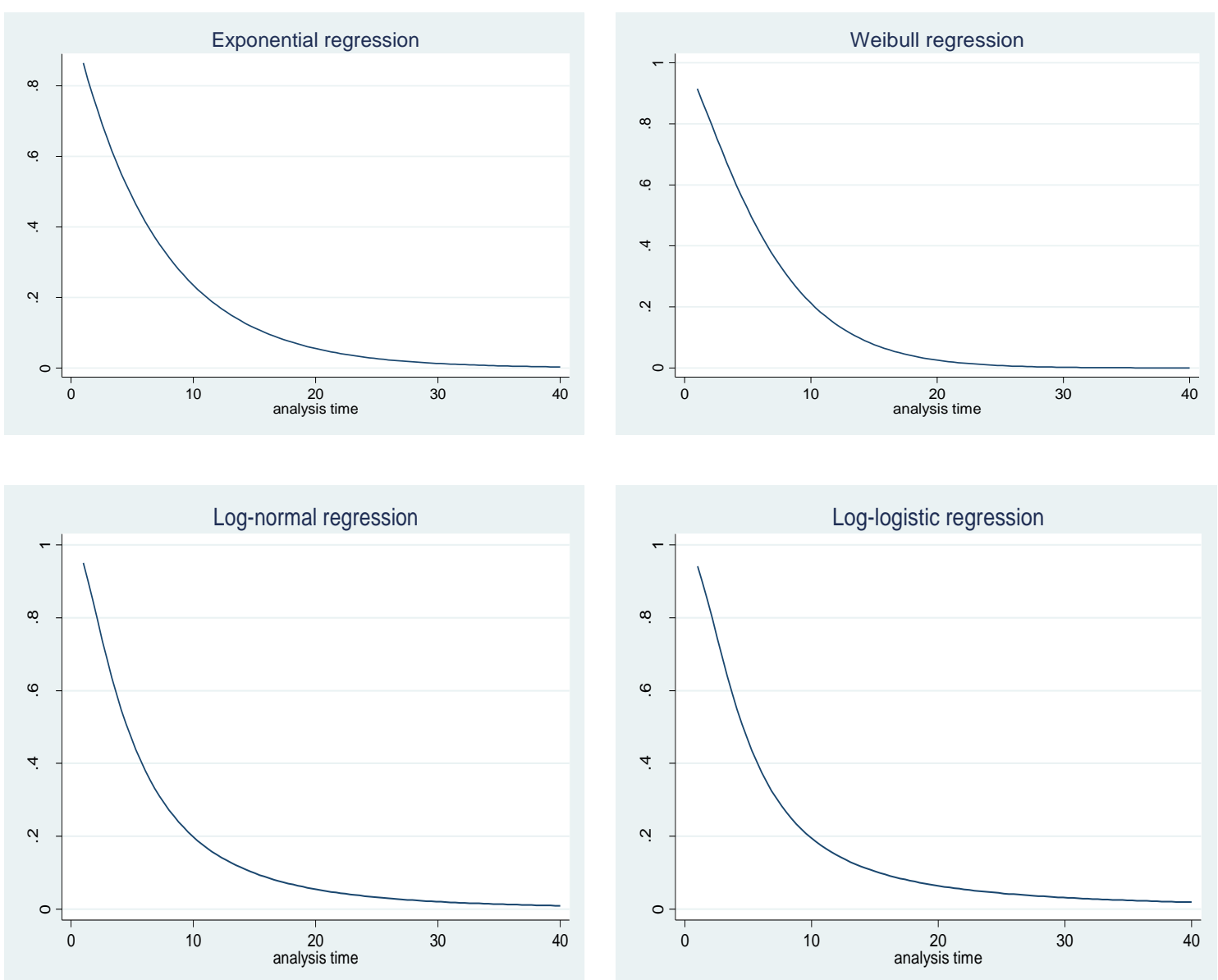

Fig. 2: Graphical Presentation for Parametric Models of Duration from Chest Pain to CAD

\section{Discussion}

The development of methods to analyze duration data has been increased from last decade (Kieding et al, 2002; Corrente et al, 2003; Morkveld, 2007). Duration analysis is referred as reliability analysis in engineering, survival analysis in biomedicine and event history analysis in sociology. The aim of the studies remained to measure event rates on 
specified duration (Lee and Wang, 2013) and to create model with covariates which affect variability of duration of event among different subjects (Cox, 1972; Hussain et al, 2011). These analyses are majorly run by two types of model, Cox proportional hazard model and accelerated failure time model. These are referred as semi-parametric and parametric duration model respectively (Gardiner, 2010; Hussain et al, 2011; Lawless, 2011). Cox regression model is widely used due to flexibility of assumptions and easy interpretation (Efron, 1977; Gardiner, 2010). Nevertheless, accelerated failure time models which are obtained from parametric distributions provide better fit in current duration studies (Oakes, 1977; Kieding et al, 2002; Morkveld, 2007; Hussain et al, 2011). Among parametric models, weibull and exponential models have been widely studied (Weibull, 1951; Moeschberger and Klein, 2003; Mingala and Estolano, 2007). On the other hand, it was found that log-normal and log-logistic also yielded goodness of fit in some studies (Gaddum, 1945; Gupta et al, 1999; Limpert et al, 2001; Orbe et al, 2002; Corrente et al, 2003; Zhou et al, 2007; Lemonte, 2014). Historically, exponential model was the first discussed duration model because of its simple statistical methods. Nevertheless, application of the model is limited to some extent because of its assumption of constant hazard function (Lawless, 2011). Weibull distribution is the extended version of exponential distribution. The distribution is reckoned to be wide applicable in the fields of manufactured items, automobile and statures of male etc. (Weibull, 1951). Akram et al also reported that weibull distribution was good fitted in their cancer data while checking by Anderson-Darling test (Akram et al, 2007). Log-logistic models are considered to be good fitted on lifetime data when the event rate gradually decreases after it reaches to a maximum point over a finite duration (Gupta et al, 1999). Log-normal distribution, on the other hand, provides good estimate when average values are low, variances are high and have positive skewed data (Limpert et al, 2001). Gaddum was the first scientist who developed log-normal distribution as an alternative to normal distribution while discovering that in nature not all data were normally distributed which was derived by de Moivre in 1753 (Gaddum, 1945). Its applications are also widely varied ranging from economics, finance, birth, geology, survival, duration, manufacturing time, stock exchange prices to biology, botany, nutrition statistics etc. (Limpert et al, 2001). Horner inferred age of Alzheimer's disease followed log-normal distribution (Horner, 1987). Similarly, Lee et al. reported that survival distribution of Hodgkin's disease and chronic leukemia were in accord with the same distribution (Lee and Wang, 2013). In accordance with these considerations, our data conformed to the literature as duration of chest pain to the diagnosis of CAD was also positively skewed distribution with average value of 5 years and maximum range reached to 40 years. Besides, there was gradual decrease after the duration of 25 years.

While comparing efficiency of Cox and parametric models, Nardi and Schemper revealed in their study parametric models yielded better results than Cox when censoring percentage does not exceed $40-50 \%$ (Nardi and Schemper, 2003). In our data, the percentage of censored observation was $26.5 \%$ which also exhibited that parametric models are good fitted for estimating duration from chronic chest pain to the diagnosis of CAD. Oakes conferred that asymptotically parametric models are well fitted if parameter values deviate far from zero (Oakes, 1977). Parameter estimates from our study also significantly far from zero while considering all three covariates. 
Our study has certain limitation as analyses were performed on secondary data. Therefore, generalization of the results may remain in quest. Though, the study was of first kind for analyzing duration from chronic chest pain to coronary artery disease. Besides, parametric model estimation was the additional excogitation in this regard.

\section{Conclusion}

The findings from the study suggested parametric modeling may also be used for predicting duration from chest pain to coronary artery disease. Log-normal accelerated failure time model is best fitted in this regard. Further studies should be derived to assess the same aspects.

\section{Acknowledgment}

The authors are indebted to principal investigator for allowing access to CADIS data. An earlier version of the paper has been presented in $11^{\text {th }}$ International Conference of Statistical Sciences, organized by Islamic Countries Society of Statistical Sciences in December 2011. The authors are also grateful to the participants for sharing their expert opinions which inscribed the study in better format.

\section{Conflict of Interest}

The authors had no conflict of interest.

\section{References}

1. Akaike H. (1974). A new look at the statistical model identification. Automatic Control, IEEE Transactions on, 19(6), 716-723.

2. Akram M., Ullah M. A., Taj R. (2007). Survival analysis of cancer patients using parametric and non-parametric approaches. Pak Vet J, 27(4), 194.

3. Al Nouzha M. M., Arafah M. R., Al-Mazrou Y. Y., Al-Maatouq M. A., Khan N. B., et al. (2004). Coronary artery disease in saudi arabia. 25(9).

4. Altman D. G., De Stavola B. L., Love S. B., Stepniewska K. A. (1995). Review of survival analyses published in cancer journals. Brit J Cancer, 72(2), 511.

5. Ata N., Karasoy D., Sözer M. T. (2008). Parametric and nonparametric survival models for nonproportional hazards. İstatistikçiler Dergisi, 1(3), 125-134.

6. Bösner S., Haasenritter J., Becker A., Karatolios K., Vaucher P., et al. (2010). Ruling out coronary artery disease in primary care: Development and validation of a simple prediction rule. Canadian Med Assoc J, 182(12),1295-1300.

7. Corrente J. E., Chalita L., Moreira J. A. (2003). Choosing between cox proportional hazards and logistic models for interval-censored data via bootstrap. J Applied Stat, 30(1),37-47.

8. Cox D. R. (1972). Regression models and life tables. J Royal Stat Soc, Series B (Methodological), 34(2), 187-220.

9. Efron B. (1977). The efficiency of cox's likelihood function for censored data. $J$ Am Stat Assoc, 72(359), 557-565.

10. Gaddum J. H. (1945). Lognormal distributions. Nature, 156(3964),463-466. 
11. Gardiner J. C. Survival analysis: Overview of parametric, nonparametric and semiparametric approaches and new developments. Proc. SAS Global Forum, 2010: Paper 252-2010. SAS Institute Inc.

12. Genders T. S. S., Hunink M. G. M. (2012). Epidemiology of coronary artery disease. Clinical Applications of Cardiac CT,3-6.

13. Gupta R. C., Akman O., Lvin S. (1999). A study of log-logistic model in survival analysis. Biometrical J, 41(4),431-443.

14. Hayat E. A., Suner A., Burak U., Dursun Ö., Orman M. N., Kİtapçioğlu G. (2010). Comparison of five survival models: Breast cancer registry data from ege university cancer research center. Turkiye Klinikleri J Med Sci, 30(5),1665-1674.

15. Horner R. D. (1987). Age at onset of alzheimer's disease: Clue to the relative importance of etiologic factors? Am J Epi, 126(3),409-414.

16. Hosmer D. W., Lameshow S. 1999. Applied survival analysis: Regression modeling of time to event data. New York: Wiley Series in Probability and Statistics.

17. Hosmer D. W., Lemeshow S., May S. 2008. Applied survival analysis: Regression modeling of time to event data. Wiley-Interscience.

18. Hussain M., Khan N. A retrospective approach in survival analysis for estimating duration from chest pain to coronary artery disease (cad). Proc. Recent Advances in Statistics, Lahore, Pakistan, 2011, 20:19: Islamic Countries Society of Statistical Sciencess.

19. Hussain M., Khan N., Uddin M. Non-parametric and parametric duration analysis of coronary artery disease with chest pain in past. Proc. Statistics for Strategic Development, Lahore, Pakistan, 2011:83: Islamic Countries Society of Statistical Sciences.

20. Kaplan E. L., Meier P. (1958). Nonparametric estimation from incomplete observations. J Am Stat Assoc, 53(457-481.

21. Kieding N., Kvist K., Hartvig H., Tvede M., Juul S. (2002). Estimating time to pregnancy from current durations in a cross-sectional sample. Biostatistics, 3(4), 565-578.

22. Kleinbaum D. G., Klein M. 2005. Survival analysis: A self-learning text. Springer Verlag.

23. Klienbaum D. G., Klien M. 2011. Survival analysis: A self learning text. New York, USA: Springer.

24. Lawless J. F. 2011. Statistical models and methods for lifetime data. John Wiley \& Sons.

25. Lee E. T., Wang J. W. 2013. Statistical methods for survival data analysis. John Wiley \& Sons.

26. Lemonte A. J. (2014). The beta log-logistic distribution. Braz J Prob Stat., 28(3),313-332.

27. Limpert E., Stahel W. A., Abbt M. (2001). Log-normal distributions across the sciences: Keys and clues. BioScience, 51(5), 341-352. 
28. Mingala M. C. V., Estolano M. P. Parametric and nonparametric survival analysis of myocardial infarction patients admitted in dr. Paulino j. Garcia memorial research and medical center in cabanatuan city. Proc. 10th National Convention on Statistics (NCS), EDSA - Shangrila Hotel, 2007.

29. Moeschberger M. L., Klein J. 2003. Survival analysis: Techniques for censored and truncated data: Statistics for biology and health. Springer.

30. Moghimi-Dehkordi B., Safaee A., Pourhoseingholi M. A., Fatemi R., Tabeie Z., Zali M. R. (2008). Statistical comparison of survival models for analysis of cancer data. Asian Pacific J Cancer Prev, 9(3).

31. Morkveld P. J. 2007. The accelerated failure time model under cross sectional sampling schemes. Thomas Stieltjes Institute for Mathematics, Almere, Netherland. 98 pp.

32. Murray C. J. L., Lopez A. D. (1996). The global burden of disease. Geneva: WHO, 270.

33. Nardi A., Schemper M. (2003). Comparing cox and parametric models in clinical studies. Stat in Med, 22(23), 3597-3610.

34. Oakes D. (1977). The asymptotic information in censored survival data. Biometrika, 64(3), 441-448.

35. Orbe J., Ferreira E., Núñez-Antón V. (2002). Comparing proportional hazards and accelerated failure time models for survival analysis. Stat in Med, 21(22),34933510.

36. Rodriguez G. 2005. Title. Lectures Notes, Princeton University. Volume:In press

37. Torpy J. M., Burke A. E., Class R. M. (2009). Coronary heart disease risk factors. J Am Stat Assoc, 302(21), 2388.

38. Weibull W. (1951). Wide applicability. J Applied Mech.

39. Zhou Y. Y., Mi J., Guo S. (2007). Estimation of parameters in logistic and loglogistic distribution with grouped data. Lifetime data analysis, 13(3), 421-429. 